Int. J. Odontostomat., 6(2):205-220, 2012.

\title{
Oclusión Dental: ¿Doctrina Mecanicista o Lógica Morfofisiológica?
}

\author{
Dental Occlusion: Mechanistic Doctrine or Morphophysiological Logical?
}

Ramirez, L. M.* \& Ballesteros, L. E.*

RAMIREZ, L. M. \& BALLESTEROS, L. E. Oclusión Dental: ¿Doctrina mecanicista o lógica morfofisiológica? Int. J. Odontostomat., 6(2):205-220, 2012.

RESUMEN: Las escuelas de oclusión han creado un constructo mecanicista que busca explicar, bajo un modelo reduccionista y determinista, una dinámica cráneo-cervical y estomatognática compleja. Estos modelos de oclusión le dedican más importancia a la morfología dental y sus geometrías interpretativas que a la morfofisiología estomatognática. La configuración musculo-esquelética cráneo-cervical tiene el poder ganado de influir concomitantemente en la ubicación espacial de la mandíbula e implícitamente en las relaciones interoclusales. La visión actual de la oclusión sigue basándose en observaciones empíricas de un siglo de antigüedad que carecen de soporte apropiado en el marco del nivel de evidencia. En la dimensión funcional los modelos de oclusión conservan los mismos vacíos que existían desde su estructuración en el siglo pasado, particularmente en sus representaciones espaciales estáticas, uniáxicas y anti-dinámicas que desestiman la individualidad de cada sujeto. Cada especialidad puede tener tantas razones como puntos de vista diferentes de "maloclusión" así como formas de tratarla.

PALABRAS CLAVE: eje cinemático de rotación, relación céntrica, oclusión bilateral balanceado, oclusión de grupo, oclusión mutuamente protegida, articulación temporomandibular, trastorno temporomandibular.

\section{INTRODUCCIÓN}

En biología, la interacción determinista (causaefecto) se da realmente por azar. Lo anterior en el marco de una multi-causalidad que no desestima probables correlaciones de eventos. La forma en que se entiende actualmente la oclusión dental no se sale de esta visión determinista y reduccionista basada en observaciones empíricas que no han sido probadas apropiadamente (Jokstad, 2012). La estreches fisiológica que representan los modelos de oclusión ponen de manifiesto las doctrinas que rigen su comprensión.

En odontología, la oclusión dental ocupa un reglón importante del entendimiento masticatorio. La mayoría de las especialidades consideran la oclusión una guía metodológica pero para otras se convierte en un dogma. Desafortunadamente, este modelo morfológico no resiste un concienzudo análisis en el marco de una visión morfofisiológica pero tampoco bajo una óptica basada en el nivel de evidencia que la soporta. Es probable que en odontología, la oclusión y su dinámica estomatognática se rija por un axioma diferente al de "la función determina la forma". Es así que el universo mecánico y geométrico en el que se mueve la oclusión, generaliza la comprensión de un funcionamiento masticatorio más real.

El objetivo de esta revisión es mostrar al lector que las doctrinas creadas sobre el tema oclusal no se apoyan en lo experimental ni se apoyan en investigaciones con criterios metodológicos bien definidos. Igualmente, que la complejidad del sistema estomatognático ha sido desestimada por estas doctrinas de oclusión dental. Una dinámica morfofisiológica completa que explique apropiadamente la relacionan entre oclusión y masticación destaca por su ausencia.

Modelos de oclusión: Referente histórico. Acomienzos del siglo pasado se inicia una carrera fascinante de ingentes esfuerzos pero igualmente de intereses hegemónicos que pautan nuestro actual entendimiento

DDS, MS, MSc. Profesor Asociado. Grupo Estomatología Biomédica, Universidad de Antioquia Medellín, Colombia.

* MD, MSc. Profesor Titular. Grupo Variaciones Anatómicas y Biomecánica Tendomuscular, Universidad Industrial de Santander, Bucaramanga-Colombia. 
en el tema del equilibrio y la estabilidad oclusal. Fueron Bonwill \& Gysi (Ash \& Ramjford, 1996; Bonwill, 1885) los pioneros en el tema oclusal, que por observación de la dinámica intermaxilar transtrusiva (movimiento mandibular de lateralidad en el plano transversal), y en usuarios de prótesis total, comienzan por sugerir un modelo conocido actualmente como "Oclusión bilateral balanceada" (que consiste en trayectorias laterotrusivas de contacto dental interarcada posterior completo compensado por contactos en balanza mediotrusivos contralaterales). Este esquema fue apoyado posteriormente por McLean (1938) en dentición natural.

Luego MacMillan (1930) modifica este modelo hacia una oclusión balanceada unilateral u "Oclusión de Grupo" (que es una laterotrusión con contacto dental interarcada posterior), basándose solo en anatomía comparada interespecies. La escuela de PankeyMann-Schuyler y Beyron entre otros apoyaban este enfoque y en el que el canino es involucrado en el esquema interdental (Mann \& Pankey, 1963; Beyron, 1964; Schuyler, 1961; Alexander, 1963; Rinchuse \& Sassouni, 1982; Nagao, 1919; Shaw, 1924;). Casi como efecto colateral a estos modelos, McCollum (Stuart \& Golden, 1981), forma en California la Sociedad Gnatológica (1926), y en la que Stuart, Stallat y Thomas (Stuart \& Golden, 1963, 1957) apoyan su ideario de relaciones dentales intermaxilares que llaman "Oclusión Orgánica".

Esta corriente de pensamiento gnatológico se sigue transmitiendo de manera residual después de su apogeo en 1980 y con muy pocas modificaciones conceptuales posteriores. La gnatología se inspira en los trabajos de D'Amico (1958) y Scaife \& Holt (1969). Utilizan el entendimiento espacial de MacMillan pero se basan en los conceptos de protección canina de D'Amico, que bajo una perspectiva morfológicaantropológica (más no fisiológica) afirman que el contacto interarcada de los caninos inhibe la actividad muscular agonista en el cierre masticatorio. Esta escuela termina por gestar el actual modelo de "Oclusión Mutuamente Protegida" (en donde los dientes anteriores guían en contacto a la mandíbula en todo recorrido excéntrico protrusivo y transtrusivo e impidiendo los contactos e interferencias en dientes posteriores) (Ash \& Ramjford; Giardot \& Redmond, 2005; Gysi, 1915; Kaplan, 1963; Reunolds, 1971; McAdam, 1976; Lucia, 1983; Schwartz, 1986; Carlsson, 2010).

Posteriormente, autores como Tipton \& Rinchuse (1991), Ahlgren \& Posselt (1963), Weinberg (1961), Yuodelis \& Mann (1965), Ingervall (1962), Rinchuse \&
Sassouni (1983), Gazit \& Lieberman (1985), Sadowsky \& Besole (1980), Sadowsky \& Polson (1984), Shefter \& McFall (1984), Egermark-Eriksson et al. (1987), deLaat \& van Steenberghe (1985), Weinberg \& Chastain (1990) y Woda (2005), muestran que la "Oclusión Bilateral Balanceada" (OBB) es la situación interarcada que se observa de forma mayoritaria en la población dentada. Matizan que la oclusión de protección canina que contiene a la oclusión mutuamente protegida (OMP) y la oclusión en grupo $(\mathrm{OG})$ raramente existen, siendo la regla general de la población actual la presencia de contactos en balanza (mediotrusivos) que acompañan bilateralmente la función de grupo laterotrusiva. Se logra evidenciar entonces que el tema oclusal ha sido polémico desde sus inicios y el análisis de su lógica funcional y su aplicabilidad clínica sigue siendo un tema actual (Begg, 1954; DeShields, 1978; Isaacson, 1976; Nelson \& Nowlin, 1955).

Teorías estáticas y uniáxicas sin evidencia. Morfofisiológicamente, la mayoría de los modelos de oclusión son solo representaciones estáticas y uniáxicas, que nacen del mas precario nivel de evidencia científica (opinión de expertos y series de casos), y no soportan apropiadamente sus conceptos. Dolwich (1983) afirma que aunque los factores oclusales pueden ser mecánicamente lógicos, estos están basados en observaciones empíricas y no han sido probados por estudios controlados (Ismail \& Bader, 2004; Rinchuse \& McMinn, 2006). Pokorny et al. (2008), en una revisión sistemática hallan 10,382 artículos relacionados con oclusión dental gnatológica, sin encontrar nivel de evidencia apropiado que soporte el modelo de esta relación dental interarcada. Errores metodológicos que involucran la falta de un diseño apropiado y otras falencias que competen a criterios de exclusión e inclusión, ausencia de grupos control y eliminación del sesgo potencial del examinador, entre otros, son frecuentes (Jokstad). No existen investigaciones en el tema de oclusión que califiquen en los niveles I, II o III de evidencia.

Los modelos de oclusión basan su conceptualización a partir de una observación morfológica y no contemplan el territorio fisiológico en sus análisis. D'Amico afirma en la introducción de un artículo clásico de oclusión que "su estudio trata principalmente con la mecánica en la oclusión de los dientes y articuladores, en lugar de factores fisiológicos y biológicos que intervienen en la función del órgano masticatorio". La escuela gnatológica cimienta todo un modelo de oclusión basado en tal declaración. A este investigador se le puede endosar la responsabilidad sobre los modelos de oclusión que se abroga la escuela gnatológica y que se apo- 
yan en un esquema de protección canina anterior. Actualmente, el grueso de investigaciones que apoyan el modelo gnatológico de protección canina con electromiografía (Shupe et al., 1984; Manns et al., 1987; Akören \& Karaagaclioglu, 1995) es menor que aquellos trabajos que con metodología similar contradicen este constructo (Dawson, 1988; Graham \& Rugh, 1988; MacDonald \& Hannam, 1984a, 1984b; Belser \& Hannam, 1985; Borromeo et al., 1995; Leiva et al., 2003; Campillo et al., 2008; Valenzuela et al., 2006; Miralles et al., 2007). Apoyados en el precepto fisiológico "la función determina la forma", no deja de ser interesante pensar en los enormes caninos de los ancestros del hombre moderno que de seguro respondían a un propósito mecánico eficiente. La especie humana podría acaso haber sobrevivido a las demandas de una dieta exigente y a ambientes hostiles con contactos intercaninos que desactivan músculos agonistas del cierre masticatorio; y consecuentemente cual sería el costo biológico de tan improductiva y utilitaria hipótesis.

Relaciones estructurales, temporales y espaciales. Los conceptos de relaciones espaciales en odontología son los más abstractos y confusos, especialmente cuando con pocos músculos masticatorios, se sostienen teorías oclusales, que desconocen todo un complejo biomecánico y neurológico significativo en la mecánica morfofisiológica de la masticación. Músculos supra e infrahioideos y músculos cráneo-cervicales (rectos y oblicuos del cuello, largos de la cabeza y cuello, semiespinales, esplenios, elevadores de la escápula, escalenos, trapecio, erectores de la espina y algunos del cinturón escapular, entre otros); influencian y son influenciados concomitantemente por una compleja dinámica estomatognática sinergista y antagonista.

Del complejo motor trigeminal, tradicionalmente en odontología solo se consideran cuatro músculos (temporal, masetero y pterigoideos) de ocho en total que inervan sus motoneuronas. Para el núcleo motor del trigémino restarían entonces suprahioideos como el milohoideo y el vientre anterior del digástrico, más algunos extraños músculos de la masticación como el tensor del tímpano y el tensor del velo del paladar que se encuentran relacionados con el oído medio y que explicarían la sintomatología referida al oído en presencia de disfunción del sistema estomatognático (Ramirez et al., 2007, 2008, 2010). Se debe reconocer que si en principio se obvian músculos putativos del sistema masticatorio, lo que resta en la comprensión de la dinámica interoclusal es poco, particularmente cuando se desestima todo un componente cráneo-cervical en su entendimiento y la influencia que este ejerce sobre la oclusión dental.
Tokiwa et al. (2008), afirman que los movimientos mandibulares funcionales como la masticación y el habla son de hecho movimientos libres dentro de un espacio funcional y no movimientos guiados por los dientes. En circunstancias normales los dientes están en contacto funcional en un rango que va de 2-7 minutos por día (Alpern, 2003) hasta de 15-40 minutos por día (Ash \& Ramjford), lo cual debate el modelo oclusal para la dinámica estomatognática. Teniendo esto en mente, se ocluye cuando se deglute, bruxa pero también en los últimos golpes antes de la deglución como lo demuestra Iwase et al. (2011), y Pameijer et al. (1969). La mayoría del tiempo la relación intermaxilar es de inoclusión postural y de libertad espacial.

Los modelos de oclusión, además de incorporar tipologías simples, estereotipadas, anti-dinámicas y uniáxicas no tienen en cuenta las condiciones exclusivas de cada individuo, lo que los hace imprácticos. Estos modelos se exhiben como estándares longevos, exentos del detrimento fisiológico y a prueba del transcurrir del tiempo. En relación a esta visión desatinada e inviable, Weiland (1994) expresa que independientemente del tipo de oclusión establecida en un paciente, ésta será transitoria y variará cuando el inevitable desgaste de los dientes se genere. Afirma que OMP con el tiempo se convierte en OG y luego en una OBB debido al constante ajuste biológico producto de la remodelación craneal, el envejecimiento y el deterioro dental (erosión, abrasión, abfracción, oxidación, atrición) (Storey, 1993). Interesantemente, la correlación entre deterioro dental y los esquemas de oclusión (incluidas las interferencias y contactos prematuros) es prácticamente nula como lo demuestra recientemente Senna et al. (2012), en donde justamente reiteran el protagonismo del deterioro normal de los dientes. Casi como un atributo, el tiempo y la biología se encargan de mostrar que hasta los modelos de oclusión y su buscada perfección tienen un conexo orden lógico a la capacidad adaptativa del natural deterioro dental.

Morfofisiología Estomatognática . La normalidad en la oclusión según Palla (2005), debe ser el resultado de la capacidad del sistema masticatorio de producir un bolo alimenticio que se deje deglutir (suave, blando y cohesivo) y que para efectos prácticos es para lo que sirve morder como fin último. Ash \& Ramjford expresan que la oclusión normal implica más que una serie de valores anatómicamente aceptables que muestren la capacidad de adaptación fisiológica y la ausencia de manifestaciones patológicas reconocibles, así como la capacidad del sistema estomatognático a adaptarse o compensar algunas desviaciones dentro del rango de 
tolerancia del sistema. Como evidencia a lo anterior se sabe que el sistema estomatognático tiene la capacidad de auto-limitarse en relación a la magnitud de reclutamiento muscular y conforme a su demanda mecanoceptiva-propioceptiva dental remanente. Los esquemas oclusales de arcos y mecanoceptiva cortos demuestran como en ausencia de molares, la dinámica fisiológica de esta contracción muscular se reduce, lo que lleva a consolidar el concepto funcional fisiológico sobre el morfológico mecanicista (Faggion, 2011; Fueki et al., 2011a, 2011b; Hattori et al., 2003; Kanno \& Carlsson, 2006; Walter et al., 2010).

Hay que recordar que la mandíbula no es un cuerpo rígido, y sus dientes en sus gonfosis igualmente sufren deformaciones conforme a las fuerzas oclusales y los ciclos de apertura y cierre que moldean transversalmente el cuerpo mandibular (contractura medial y bilateral del haz inferior del músculo pterigoideo lateral en apertura). Por si fuera poco, eso sucede con una mínima parte de toda la máxima capacidad masticatoria y por lo general con un patrón interoclusal uni o bilateral que depende de qué se este macerando, de la ubicación espacial del cráneo durante este proceso y también de la etapa de la maceración en la que se encuentre.

Al margen de la trayectoria de los movimientos de apertura y cierre mandibular, los modelos de oclusión explican las relaciones dentales interarcada en solo tres escuetos movimientos extremos (protrusión, mediotrusion y laterotrusion), y que solo son representados en tres ejes (sagital, coronal y transversal). Una serie de dogmas (entre muchos más) nacen de esta precaria relación espacial y demuestran como las escuelas de oclusión consideran (crédulamente) que la forma determina la funcion (Carlsson):

Los cóndilos deben descansar en su posición más antero-superior contra la cara posterior de la eminencia articular.

El disco articular debe estar apropiadamente interpuesto entre los cóndilos y las fosas mandibulares.

Los dientes posteriores deben tener contacto parejo y simultaneo en posición céntrica.

Los dientes anteriores deben contactar y desocluir a los dientes posteriores en movimientos excéntricos.

En posición vertical de la cabeza, los dientes posteriores deben contactar mas prominentemente que los contactos de los dientes anteriores.

Los dientes anteriores deben proveer una guía desoclusiva con trayectorias superficiales que desocluyan los dientes posteriores.
Koolstra (2002) afirma que el análisis de la dinámica de movimientos musculo-esqueléticos el análisis debe contar con toda una maquinaria de elementos biome-cánicos para su comprensión que van más allá de simples planos espaciales y que incluyen entre muchas mas: la forma del objeto, posición, velocidad, aceleración lineal y angular, planos cartesianos $\mathrm{X}, \mathrm{Y}, \mathrm{Z}$, zenit, elevación y giro de orientación, ubicación espacial, masa, tiempo, torque, distancia, centro de gravedad. Contrastantemente, los únicos tres movimientos con los que se construye un modelo oclusivo, dependen de una geometría arbitraria construida desde una hegemonía morfológica doctrinaria que desestima la infinita posibilidad espacial fisiológica de vectores intermedios entre ellos y que se pueden ver trazados entre el cóndilo mandibular y la base del cráneo pero asimismo sobre la morfología dental, sin ser la forma oclusal de los dientes, óbice de una libertad espacial para movimientos interdentales que sobrepasan sus fronteras cuspideas mayormente cuando son determinados por una compleja dinámica cráneo-cervical.

La relación cráneo-cervico-mandibular esta intrincadamente relacionada con la posición intermaxilar e implícitamente con la oclusión. Esta relación intermaxilar no es una posición estable ni única y es modificada por la actividad dinámica de su ambiente perioral y gravitacional (Mohamed \& Christensen, 1985; Darling, 1984; Goldstein, 1984; Manfredini et al., 2012). La única función antigravitacional que se le endosa a los músculos vecinos de cabeza y cuello en la zona cervical desestima la correspondencia de estos y los músculos de la masticación en las relaciones interarcada, y más aun, la influencia de reciprocidad dinámica que tiene la posición interarcada en la postura del cuerpo en el espacio (Funakosh, 1976; McLean, 1973; Rocabado, 1982-1983). Contracciones aisladas y combinadas de este complejo engranaje musculo-esquelético cráneocervical se reflejan en múltiples posiciones interarcada que modifican las posiciones interoclusales del ciclo masticatorio, la trayectoria del cierre mandibular, la posición postural y la capacidad contractora muscular (Manns \& Diaz, 1988; Wallace, 1993; Pullinger \& Seligman, 2000; Schellhas \& Keck, 1989; Mongini et al., 1989; Schellas, 1989; Cooper et al., 1986). El cráneo necesita ser estabilizado a través de su base y el hueso hioides para ofrecer a la mandíbula un marco relativamente estable a través de una musculatura distante a la mandíbula y que corresponde a la del cuello y la espalda que actúan como retenedores posturales en una dinámica cráneo-cervico-mandibular recíproca, e intrincada (Dawson, 1988, 1995; Hidaka \& Iwasaki, 1999; Dos Santos, 1991; Williamson \& Lundquist, 1983). 
El cuerpo mandibular puede desplazarse conforme a la dinámica del ambiente perioral cráneo-cervical y ocurre debido a la libertad de movimientos que permite la articulación temporomandibular (ATM). Según Koolstra, las superficies articulares de la ATM son altamente incongruentes y separadas en el espacio (acople desigual cóndilo y hueso temporal), lo que permite un gran rango de libertad entre ambos huesos cuando se ponen en contacto biomecánico durante el movimiento. Afirma que el disco articular reduce la incongruencia y mejora el contacto interóseo que se ve reflejado en mayor estabilidad, sin embargo no se logra una estabilidad absoluta, que genera una cinemática de redundancia. La condición deformable del disco y las amplias fronteras óseas permiten varios grados de libertad (Ej., Bennett inmediato y céntrica larga) (Preiskel, 1971). Adicionalmente, la laxitud del los ligamentos que contiene este complejo cóndilo-disco-temporal no limita los movimientos a ejes rígidos y fijos y antes por el contrario permite trayectorias articulares e interdentales infinitas.

Adicionalmente, el rango de movimientos que permiten los ligamentos de la ATM sobrepasa por mucho el ancho de la tabla oclusal o la relación interincisal interarcada, situación que desmantela cualquier modelo oclusal en que los movimientos en el plano transversal deben idealmente (en teoría) llegar hasta la frontera del contacto interdental. Concebir los determinantes de la morfología dental es factible cuando solo se cuenta con tres estrictos, rectilíneos y bordeantes movimientos en el plano transversal, pero desde luego es mas complejo cuando se cuenta con todos los planos cartesianos del movimiento espacial de la ATM y no se desestima la amplitud y laxitud en el rango de movimiento permitido por los ligamentos de la ATM.

El eje lógico del que parte la configuración de toda esta analítica vectorial oclusal, depende del dogma espacial mas renombrado y polémico: "Relación céntrica"; concepto que se ha modificado sustancialmente en siete oportunidades hasta la actual octava versión del glosario de términos prostodónticos. En esta visión y sin entrar en muchos detalles, se pretende que el complejo cóndilo/disco/fosa mandibular/tubérculo articular, pasen de una situación ortopédica mecánicamente neutral y libre (compuesta por rangos de movimiento), a una ubicación forzada, puntual, rígida e innegociable, decretada por las escuelas de oclusión (Keshvad \& Winstanley, 2001; McKee, 2005; Alexander et al., 1993). Esta anquilosada visión de una relación interósea articular cuenta con la ausencia de anclas anatómicas que la sostengan espacialmente, como los ligamentos capsular, temporomandibular, estilo-mandibular, esfenoman- dibular y que se encuentran laxos en esta posición ortopédica muscular; siendo estos últimos (estructuras visco-elásticas y dinámicas), los que ponderan la ubicación de los cóndilos de la ATM. Los modelos de las escuelas de oclusión posteriormente conectan estos puntos "céntricos" de cada ATM, y entre cóndilos asimétricos y nada paralelos, con un eje paralelo imaginario que lo denominan "eje cinemático de rotación" o supuestamente "eje de bisagra", concepto que tiene casi un siglo de creado sin modificaciones ni muchos cuestionamientos (Winstanley, 1985; Starcke, 2001, 2002a). El principio físico de una bisagra, es el movimiento en torque de un cuerpo con un eje de rotación paralelo, situación que por características morfológicas estáticas y también dinámicas, no se cumple de manera estricta entre los cóndilos de la mandíbula. Entonces el "eje cinemático de rotación" seria el promedio entre dos o mas ejes lo que evidentemente lo hace "imaginario" por lo que un giro puro y sostenido en movimientos rotatorios y translatorios bajo estas circunstancias no ocurre sino en la imaginación (Hellsing et al., 1995; Piehslinger et al., 1994; Tsao, 1986; Simpson et al., 1984; Schulte et al., 1984). Kinderknecht et al. (1992), encuentran que la posición de eje cinemático de rotación varia en sujetos saludables cuando los dientes son repetidamente ubicados en relación céntrica.

A la sazón, hacer que la oclusión coincida micrométricamente con posiciones y ejes espacialmente generosos, que cambian conforme se modifica la posición del cráneo sobre las vértebras cervicales, durante apertura y cierre con dientes en sus gonfosis, solo puede ser explicado por una visión determinista y mecanicista oclusal. Visión que pretende además registrar y simular en una sola posición (como deteniendo el tiempo), estas relaciones ideales en un simulador mecánico (articulador) construido con medidas promedio y algunos determinantes arbitrarios como una pared medial irreal y trayectorias rectilíneas y formateadas que anatómicamente no existen (Starcke \& Engelmeier, 2006; Starcke, 2002c, 2002c, 2002d, 2003).

La masticación es un acto estereotipado subconsciente según Masserman (1963). Cuando a las personas se les pide que registren movimientos de la mandíbula en un rodete de cera para llevar el registro interoclusal obtenido a un articulador, el paciente se confunde al hacer consciente (en la corteza cerebral), un movimiento automático y estereotipado (irreflexivo y subconsciente originado en los ganglios basales y zonas reticulares del tronco encefálico). Este investigador lo compara con detener a una persona caminando, y pedirle que muestre cómo camina, afirman- 
do que tiene el mismo efecto: será una imitación torpe de su andar natural. De esta forma sostiene que los movimientos mandibulares son solo una pantomima de imitación en tres ejes de los verdaderos movimientos funcionales. Con esto en mente, el registro interoclusal es un error y los resultados sólo son un sofisma de tratamiento ya que los movimientos espontáneos de la masticación no se pueden restringir a un potpurrí de reglas anti-fisiológicas acuñadas en modelos de oclusión (Jokstad, 2012).

Referente evolutivo. Antes de la aparición de los primeros peces con mandíbulas (gnatostomos) en la era paleozoica temprana hace aproximadamente 416-444 millones de años, el proceso de alimentación por un antro oral no necesitaba el desglose biomecánico de alimentos ni un proceso parecido al dentellar que se realiza entre dos maxilares con un hueso móvil que corresponde a la mandíbula y del que nuestros ancestros primates, hace 65 millones de años, ya empleaban. De los casi 58.000 vertebrados actuales, mas de $99 \%$ tienen mandíbulas (Anderson et al., 2011). Las bocas sin mandíbulas (agnatos) correspondían a cefalocordados y ciclóstomos, de los cuales aun sobreviven las lampreas. Evolutivamente los peces con mandíbula correspondieron a una nueva etapa evolutiva (seláceos, bradiodontos, condrósteos y teleósteos) que les permiten además de una alimentación más rica y variada, la posibilidad de presas de mayor tamaño, sin dejar de lado la posibilidad de otras funciones intermaxilares como el ataque, la defensa y un efectivo aprisionamiento de la presa (enormes caninos) (Brazeau, 2009). Con la mandíbula presente, debe ponerse igualmente en consideración el desarrollo de una comunicación efectiva que involucraba movimientos intermaxilares en los procesos de articulación labio-dento-palato-lingual para la producción de fonemas en la emisión de un lenguaje cargado de sintaxis y mensaje. Por último, los maxilares contiene dientes que en el mar fueron escamas (con iguales capas de esmalte, dentina y cemento) y que rodeaban el esfínter oral. Estas estructuras blanquecinas y esmaltadas juegan un rol importante en el cortejo y la estética en la raza humana. La forma dento-musculo-esquelética es determinada por la alimentación (función), que juega un rol importante en la morfometría dental y maxilar (forma) y que explica la variada presentación de formas dentales interespecies que se observan actualmente. Con esto en mente, el tipo de ingesta y su tamaño(herbívora, carnívora, omnívora), la dieta (dura, blanda, fluida), el procesado (bolo macerado y cohesivo o bolo entero) y la complejidad del tracto digestivo complementario en zonas caudales a la boca (primera etapa de este proceso estomacal), juegan todos un rol determinante en las ca- racterísticas morfológicas de dientes y maxilares. La forma dental no es producto de la dimensión imaginativa, creativa y estética de un solo individuo, sino de toda una marca evolutiva que solo responde a las necesidades funcionales de sus ancestros. Riñendo con este precedente, Peter K. Thomas y colaboradores, fabrican "por mandato" un patrón morfológico dental que determina (según ellos) la forma oclusal humana ideal intra e interarcada, sin investigaciones que sustenten y se basen al menos en estudios de variaciones morfológicas dentales previas y en el marco de un espíritu por de más creacionista (Mohl, 1988).

Masticación y morfología: función y forma. Dependiendo de lo que se come, la ingesta del alimento se somete a un proceso de corte y/o trituración en el que la comida entra en un proceso de destrucción invariable y antepuesta a la deglución. En presencia de saliva la comida se conforma en un cuerpo laxo, pegajoso y manejable que adquiere propiedades reológicas al fluir y ser repetidamente deformado entre superficies duras (dientes) y blandas (lengua, paladar, músculos bucinador, orbicular de los labios, palatogloso y palatofaringeo), en un ciclo sistemático. Las estructuras blandas se encargan de impulsar el bolo aplastado en cada golpe masticatorio y desde los pliegues orales hacia una zona neutra a la que invariablemente llega y que corresponde al espacio de oclusión interdental en el que nuevamente es aplastada y molida la comida de manera fuerte (Figura 1). Tokiwa et al., expresan que todos los conceptos de oclusión se deben basar en movimientos de molienda fuerte como el bruxismo.

Bajo una comprensión reológica y sin muchas pretensiones mecanicistas y geométricas, se logra ver el parecido de la morfología dental al de la geografía de zonas montañosas, en las que se notan sobresalientes filosas, prominentes relieves y profundas depresiones en los que el caudal de los ríos corre, ajustándose a un principio de la mecánica de esfuerzo y deformación de fluidos que los hace recorrer sitios de menor resistencia entre estos relieves. Nos cuestionamos si podrían las formas dentales responder solo a un simple principio reológico en el que sus rebordes, lomos, surcos principales y accesorios, vertientes y contactos interproximales corresponden a formas que facilitan la molienda y evacuación interdental eficiente de un bolo alimenticio repetidamente aplastado por los ciclos masticatorios y anterior a su deglución.

Si no fuese así de simple, las oclusiones asociadas a situaciones impensables según los modelos de oclusión y eficiencia masticatoria no permitirían la ingesta 


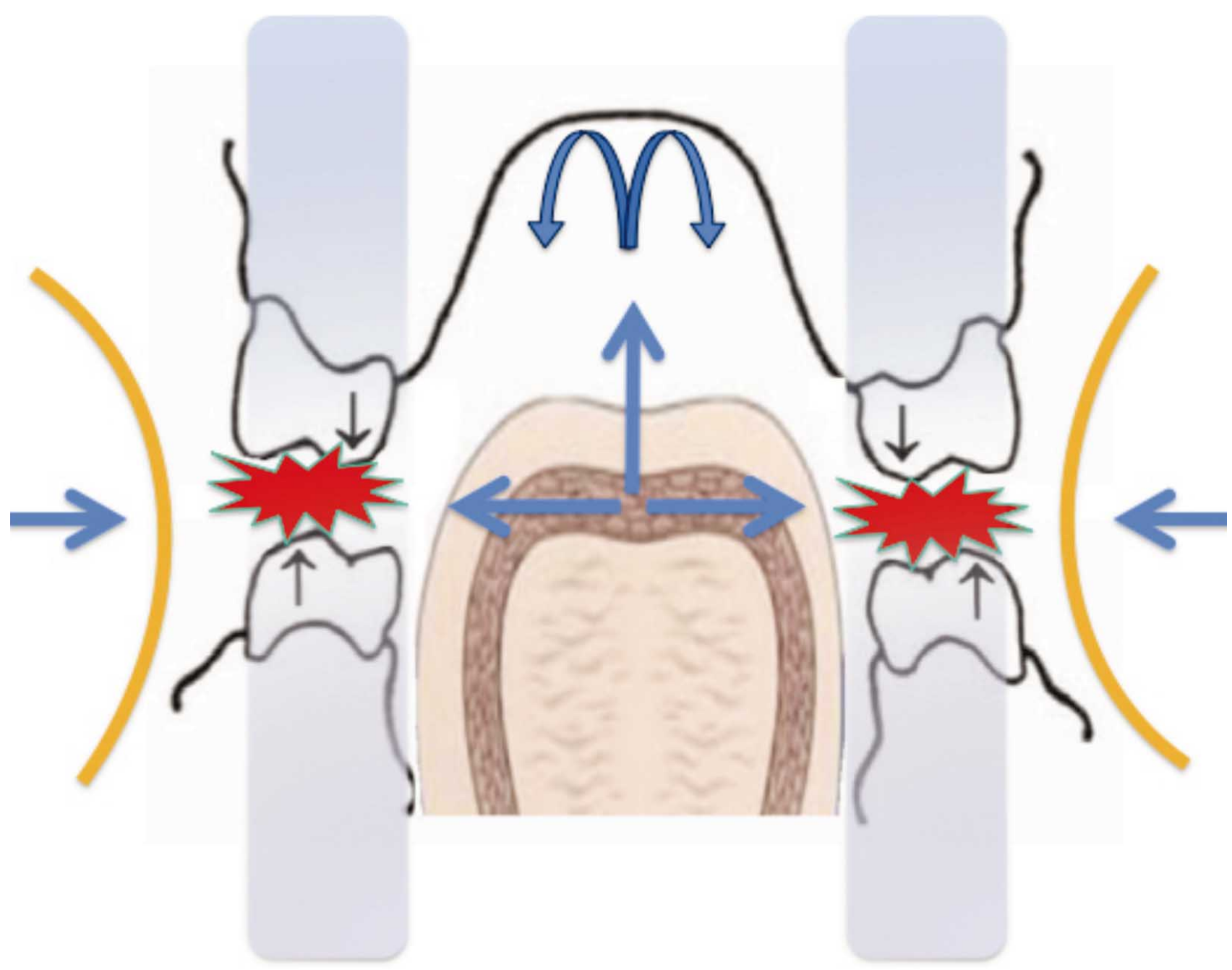

Fig. 1. Esquema de la masticación. Proceso de corte y/o trituración. En presencia de saliva la comida se conforma en un cuerpo laxo, pegajoso y manejable que adquiere propiedades reológicas al fluir y ser repetidamente deformado entre superficies duras (dientes) y blandas (lengua, paladar, músculos bucinador, orbicular de los labios, palatogloso y palatofaringeo), en un ciclo sistemático. Las estructuras blandas se encargan de impulsar el bolo aplastado en cada golpe masticatorio y desde los pliegues orales hacia una zona neutra (color púrpura) a la que invariablemente llega y que corresponde al espacio de oclusión interdental en el que nuevamente es aplastada y molida la comida de manera fuerte.

calorica en personas con: retrognatismo severo, hiper/ hipodivergenia facial, prognatismo, edentulismo parcial, asimetría facial, hiper/hipoplasia condilar, mordidas cruzadas, abiertas o profundas, macro-micrognatismo maxilar, amelo/dentinogénesis imperfecta, entre otros; pero las funciones estomatognáticas en estos casos se adptan y funcionan.

Oclusión y bruxismo: factor de riesgo y protector . Los factores de riesgo biológico son condiciones que se asocian con una mayor morbi-mortalidad, en este caso del sistema estomatognático. Contrastantemente, los factores que previenen o disminuyen los efectos de cau- sas nocivas son factores protectores, que ayudan a la adaptación del individuo al ambiente.

Cuando sucede el bruxismo durante los ciclos del sueño y estados transicionales, este desorden de movimiento rítmico es una poderosa parasomnia motora y es clasificada como un trastorno de excitación y vigilia del sueño. Esta actividad disfuncional inconsciente tanto en estados de vigilia como de sueño, adquiere alcances patológicos por su capacidad deletérea en el sistema estomatognático. La fuerza interoclusal nocturna reportada en bruxomanos esta en un rango de 153-796,3 Newtons con una duración media de 7,1 +/- 5,3 segun- 
dos (8-14 veces por hora); y hasta 14 veces por encima del máximo voluntario electromiográfico (Miles et al., 2004; Clarke et al., 1984; Ware \& Rugh, 1988; Lavigne et al., 2001). Conforme a lo anterior, el bruxismo cumple a cabalidad los estándares de factor de riesgo para el sistema masticatorio, sin embargo, éste paradójicamente muestra tener propiedades homeostáticas de orden sistémico, con implicaciones hemodinámicas, mnemonisticas y cognitivas (van der Bilt, 2011; Koizumi et al., 2011; Miyake et al., 2012; Ono et al., 2010).

Es así que el bruxismo, en el que se aprieta y rechina las superficies oclusales, parece ser una actividad masticatoria de doble fin con efectos beneficiosos ya que llega a fungir de factor protector en los procesos de memorización y en la actividad nerviosa autónoma del cuerpo (Sato et al., 2010; Ono et al., 2011; Okada et al., 2007; Miyake et al., 2008; Lee et al., 2008). Bruxar suprime las respuestas endocrinas al estrés en el eje hipotálamohipofisiario-suprarrenal que activado desencadena un tono simpático elevado generando como respuesta la presencia de catecolaminas y corticotropinas endógenas. El sistema vegetativo responde a un balance simpáticoparasimpático en el que el tono automático de gasto y/o ahorro de energía se ve expresado en conductas automáticas del cuerpo. Estas réplicas autónomas y fisiológicas son normales, sin embargo, en estados disautonómicos (generados por la ansiedad), los elevados niveles de la hormona corticotropina (cortisol), puede convertirse en un mecanismo de consumo de energía corporal malsano con efectos deletéreos sobre el hueso, cartílago, músculos y sistema nervioso cuando no se degrada o disminuye a un nivel aceptable. Por lo visto, bruxar parece ser un mecanismo comportamental útil para lidiar con el estrés que también incrementa la expresión del receptor de glucocorticoides en el hipocampo (lóbulo temporal), potenciando la memoria de trabajo pero igualmente la respuesta histaminérgica en el mismo locus desde el núcleo mesencefálico trigeminal.

Naturalmente, la dimensión emocional del bruxismo además de ejercer un efecto motor corporal general y primario expresado con contracturas isotónicas $\mathrm{e}$ isométricas repetidas y dañinas al sistema masticatorio, tiene un efecto contrario y secundario al balancear el tono simpático que inicia estos episodios como respuesta de estados simpatéticamente sostenidos. Esta amplia, contrastante e intrincada conectividad a multinivel entre el sistema límbico es el modulador mas importante de la hemostasia del sistema nervioso central que es capaz de modificar el equilibrio corporal como respuesta a un estimulo de supervivencia (lida et al., 2007; Tanaka et al., 2008; Kato \& Rompre, 2011).
Observaciones finales. Endosarle a una relación dental interarcada no ideal (maloclusión), el origen de patologías y disfunciones estomatognáticas es imprudente, particularmente sin evidencia de tal causalidad. Vista la oclusión desde un único modelo ideal estético y morfométrico basado en arquetipos estrictos y excluyentes de la variedad, es una visión absolutista en biología. Lo que no está incluido en estas categorías, es considerado una maloclusión (Angle, 1899, 1907; Andrews, 1972). Hasta ahora no se ha identificado un patrón oclusal o masticatorio especifico más óptimo. Behrents (1986) afirma que se pueden encontrar personas con sistemas masticatorios funcionalmente óptimos con relaciones interoclusales comprometidas y desorganizadas (maloclusiones para el ideal del modelo) y contrariamente encontrar oclusiones perfectas con sistemas masticatorios trastornados y malestar musculoesquelético activo.

Si las relaciones intra e interarcada se consideran de base como maloclusiones, estas deben generar patología o por lo menos impedimentos funcionales. Sobre este tópico no hay evidencia científica que corrobore de manera seria tal afirmación. Normal, implica que se encuentran en ausencia de enfermedad, y normal en un sistema biológico es dado con un rango fisiológico de adaptación según Ash \& Ramjford. En la población general la oclusión ideal es una excepción y la mayoría de las personas se encuentran en maloclusion. Palla afirma que la oclusión ideal es simplemente un constructo teórico creado por odontólogos con la intención de simplificar la parte técnica del trabajo especialista; y que la oclusión ideal no es sinónimo de oclusión fisiológicamente correcta. Nosotros creemos al igual que Palla, que el termino "maloclusion" debe eliminarse de la semántica odontológica. Pensamos que el prefijo "mal" en cualquier condición interoclusal, deja implícito un mal funcionamiento que probablemente no sea real y que clasifica cualquier condición clínica como patológica. Si términos como mala-audición, mala-locomoción, maladigestión y mala-visión entre otros, antecedieran cualquier criterio diagnostico en protocolos de clasificación para cada disciplina en salud, lo mas seguro es que la población entera estaría enferma y sin estarlo.

Ash \& Ramjford afirman que la retorica maloclusiva es tanto cándida como peligrosa; y en concordancia Rinchuse et al. (2007), afirman que la meta "a todo costo" de alcanzar oclusiones ideales es injustificada y posiblemente iatrogénica. McNamara et al. (1995), creen que aunque una oclusión estable es un tratamiento de ortodoncia razonablemente objetivo (eliminar graves discrepancias oclusales), el no logro de 
un ideal gnatológico específico no da lugar a signos y síntomas del sistema estomatognático. La oclusión, las interferencias y contactos prematuros asociados, ya no siguen siendo considerados factores primarios ni únicos en la naturaleza multifactorial de los trastornos temporomandibulares (TTM), con evidencia importante que lo demuestra (Manfredini et al., 2011; Michelotti et al., 2005; Le Bell et al., 2002). Türp et al. (2008), afirman que para pacientes que tengan la mala suerte de presentar dolor y disfunción en el sistema masticatorio, existe una alta probabilidad de que sus problemas se analizarán y tratarán dentro de algún paradigma oclusal.

Modificar la oclusión dental a través de sofisticados tratamientos invasivos tiene implicaciones serias y efectos colaterales que no influencian una dimensión morfofisiológica saludable y real en la dinámica estomatognática. Lo señalan Throckmorton et al. (2001), al demostrar que los ciclos masticatorios antes y después de un tratamiento ortodontico son idénticos. Koyano et al. (2012) afirman que igual que una prótesis dental, una prótesis ocular es morfológicamente correcta y tiene la estética adecuada, pero no puede mejorar la función sensorial ni motora de la estructura reemplazada. Rinchuse et al., en una amplia revisión del tema oclusivo dental no pueden encontrar diferencias entre pacientes ortodonticos tratados bajo un enfoque gnatológico puro y aquellos que no, y tampoco en aquellos con maloclusiones sin tratamiento. En el mismo sentido, la presencia de TTM entre pacientes con maloclusiones sin ortodoncia y pacientes postortodonticos fue igual. Matizan que una "oclusión ideal" no ha probado disminuir un TTM, ocupando un rol insignificante o nulo en su multifactorialidad. En el mismo tenor, López-Garavito et al. (1985), no encuentran diferencias de estabilidad entre pacientes con mordida abierta anterior (y sin contacto canino) y aquellos con oclusiones ideales. Por ultimo, Bush (1985) muestra como el modelo de oclusión canina no previene el TTM en 300 estudiantes con guía canina ideal.

Cada especialidad puede tener tantas razones como puntos de vista diferentes de maloclusión, así como formas de tratarla (o quizá sobretratarla): el rehabilitador oral puede endosarle su responsabilidad a la estabilidad, el ortodoncista a la guía canina, el prostodoncista a la dimensión vertical, el periodoncista a la higiene por apiñamiento, el cirujano maxilofacial a las relaciones transversales intermaxilares, el endodoncista al contacto prematuro y las interferencias, el odontopediatra a la erupción y llaves de oclusión, el fonoaudiólogo a la articulación del lenguaje, el otorrino a la respiración oral y la obstrucción aérea, el gastroenterólogo a la primera fase de la digestión, entre otras. En concreto lo que se debe aspirar es a no sobrevalorar la variable oclusiva en una multicausalidad, particularmente cuando no se cuenta con soporte científico ni algún nivel evidencia serio que avale cualquiera de las rutas terapéuticas.

Sería prudente cuestionarse si es entonces factible concebir la compleja dinámica estomatognática bajo constructos reduccionistas, imprácticos y anti-fisiológicos enmarcados en tres solitarios movimientos espaciales y soportados en teorías de oclusión uniáxicas y estáticas. En torno al tema de la oclusión, sus modelos y su alcanzado soporte científico en el tema del nivel de evidencia, la máxima de Isaacs \& Fitzgerald (1999) ayuda a percibir bajo otra perspectiva esta realidad odontológica. Afirmaban "... .algunos autores recomiendan volver a las practicas pasadas y desarrollar alguna de las alternativas a la odontología basada en la evidencia, como la practica basada en la eminencia, en la vehemencia o en casos extremos, en la providencia...".

Nos preguntamos si tiene sentido adherirse a dogmas fundamentalistas de oclusión cuando la tendencia mundial actual se representa bajo un modelo más pragmático y parecido a "verdades en construcción". Visión que le da espacio para maniobrar a lo biológico y lo morfo-fisiológico y desestima las doctrinas y el determinismo de estas que solo son decretos impuestos y conceptos vagos y ambiguos sin algún nivel de evidencia que los sustenten. Los modelos de oclusión (todos) no explican la compleja dinámica de un sistema estomatognático funcional y mucho menos uno en estado disfuncional (National Institutes of Health Technology Assessment Conference Statement, 1996; Ohrbach et al., 2010; Dawson, 1999). Isaacson afirmaba que muchos tipos de oclusión ajenos a los conocidos, pueden ser asimismo biológicos y fisiológicos en cada paciente ya que un solo arquetipo de oclusión no es funcional ni aplicable en todos.

Las escuelas de oclusión hasta ahora no perciben la multifactorialidad de eventos que la acompañan, tampoco su individualidad, menos su concomitancia y reciprocidad con elementos como la postura cervical y corporal, y su relación con un balance sistémico intrincado, entre otros co-factores agregados. Es imperante que se repiensen las disciplinas, pero más significativo que no se sigan viendo como doctrinas y se basen en los actuales dominios tecnológicos y biológicos que están sufriendo cambios muy rápidos. Gradualmente, las escuelas odontológicas formadas a partir de conceptos individualistas e inviables de 
oclusión, están destinadas a ceder en cambios conceptuales concernientes a la eficiencia masticatoria. Se destaca la necesidad de entender las relaciones interdentales desde una perspectiva que oriente el futuro clínico en una dimensión fresca y en la que la perspectiva molecular, neurofisiológica, fisiopatológica y morfofisiológica sean los principales intérpretes de la conceptualización de un sistema estomatognático altamente complicado y poderosamente articulado con el resto del cuerpo.

RAMIREZ, L. M. \& BALLESTEROS, L. E. Dental occlusion: mechanistic doctrine or morphophysiological logical? nt. J. Odontostomat., 6(2):205-220, 2012.

ABSTRACT: Occlusion schools have created a mechanistic construct that seeks to explain, under a reductionist and deterministic model, a craneal-cervical and stomatognathic complex dynamic. These occlusion models dedicate more importance to dental morphology and its interpretative geometries than a stomatognathic morphophysiology. Craneocervical musculoskeletal configuration has the gained power to concomitantly influence the jaw location in the space and implicitly in the interocclusal relations. The current vision of the occlusion is based on empirical observations of almost a century that lack support in the level of evidence framework. In the functional dimension occlusion models maintain the same gaps that existed from its origins since the last century, particularly in its static, uniaxic and anti-dynamic spatial representations that dismiss the individuality of each subject. Each specialty can have as many reasons as different points of view about "malocclusion" as well as techniques to treat it.

KEY WORDS: terminal hinge axis, centric relation, bilateral occlusion, group occlusion, mutually protected occlusion, temporomandibular joint, temporomandibular disorder.

\section{REFERENCIAS BIBLIOGRÁFICAS}

Akören, A. C. \& Karaagaclioglu, L. Comparison of the electromyographic activity of individuals with canine guidance and group function occlusion. J. Oral Rehabil., 22:73-7, 1995.

Ahlgren, J. \& Posselt, V. Need of functional analysis and selective grinding in orthodontics. Acta Odontol. Scand., 25:3-13, 1963.

Alexander, P. C. Analysis of the cuspid protected occlusion. J. Prosthet. Dent., 13:309-17, 1963.

Alexander, S. R.; Moore, R. N. \& DuBois, L. M. Mandibular condyle position: comparison of articulator mountings and magnetic resonance imaging. Am. J. Orthod. Dentofacial Orthop., 104:230-9, 1993.

Alpern, M. C. The ortho evolution-the science and principles behind fixed/functional/splint orthodontics. New York, GAC International, 2003.

Andrews, L. F. The six keys to normal occlusion. Am. J. Orthod., 62:296-309, 1972.

Anderson, P. S.; Friedman, M.; Brazeau, M. D. \& Rayfield, E. J. Initial radiation of jaws demonstrated stability despite faunal and environmental change. Nature, 476:206-9, 2011.
Angle, E. H. Classification of malocclusion. Dent. Cosmos, 41:246-64, 1899.

Angle, E. H. Malocclusion of teeth. $7^{\text {th }}$ ed. Philadelphia, SS White Dental Mfg. Co., 1907.

Ash, M. M. \& Ramjford, S. Occlusion. $4^{\text {th }}$ ed. Philadelphia, Saunders, 1996.

Begg, P. R. Stone Age man's dentition. Am. J. Orthod., 40:298-312, 1954.

Behrents, R. JCO interviews Dr. Rolf Behrents on adult craniofacial growth. J. Clin. Orthod., 20:842-7, 1986.

Belser, U. C. \& Hannam, A. G. The influence of altered working-side occlusal guidance on masticatory muscles and related jaw movement. J. Prosthet. Dent., 53:406-13, 1985.

Beyron, $\mathrm{H}$. Occlusal relation and mastication in Australian aborigines. Acta Odontol. Scand., 22:597-608, 1964.

Bonwill, W. G. Geometrical and mechanical laws of articulation. Anatomical articulation. Transactions of the Pennsylvania Odontological Society, 1885.

Borromeo, G. L.; Suvinen, T. I. \& Reade, P. C. A comparison of the effects of group function and 
canine guidance interocclusal device on masseter muscle electromyographic activity in normal subjects. J. Prosthet. Dent., 74:174-80, 1995.

Brazeau, M. D. The braincase and jaws of a Devonian 'acanthodian' and modern gnathostome origins. Nature, 457:305-8, 2009.

Bush, F. M. Malocclusion, masticatory muscle, and temporomandibular joint tenderness. J. Dent. Res., 64:129-33, 1985.

Campillo, M. J.; Miralles, R.; Santander, H.; Valenzuela, S.; Fresno, M. J.; Fuentes, A. \& Zúñiga, C. Influence of laterotrusive occlusal scheme on bilateral masseter EMG activity during clenching and grinding. Cranio, 26:263-73, 2008.

Carlsson, G. E. Some dogmas related to prosthodontics, temporomandibular disorders and occlusion. Acta Odontol. Scand., 68:313-22, 2010.

Clarke, N. G.; Townsend, G. C. \& Carey, S. F. Bruxing patterns in man during sleep. J. Oral Rehabil., 11:123-37, 1984.

Cooper, B. C.; Alleva, M.; Cooper, D. L. \& Lucente, F. E. Myofacial pain dysfunction: Analysis of 476 patients. Laryngoscope, 96:1099-106, 1986.

D'Amico, A. The canine teeth: normal functional relation of the natural teeth of man. J. S. Calif. Dent. Assoc., 26:6-23, 1958.

Darling, D. W. Relationship of head posture and the rest position of the mandible. J. Prosthet. Dent., 52:111-5, 1984.

Dawson, P. E. Evaluation, diagnosis and treatment of occlusal problems. $2^{\text {nd }}$ Ed. St Louis, Mosby Co., 1988.

Dawson, P. E. New definition for relating occlusion to varying conditions of the temporomandibular joint. J. Prosthet. Dent., 74:619-27, 1995.

Dawson, P. E. Position paper regarding diagnosis, management, and treatment of temporomandibular disorders. The American Equilibration Society. J. Prosthet. Dent., 81:174-8, 1999.

de Laat, A. \& van Steenberghe, D. Occlusal relationships and temporomandibular joint dysfunction. Part I: epidemiologic findings. J. Prosthet. Dent., 54:835-42, 1985.

DeShields, R. W. Gnathological considerations of a controversial nature. Dent. Surv., 54:12-8, 1978.

Dolwich, F. W. Diagnosis and etiology of internal derangements of the temporomandibular joint. In: Griffiths, R. H. (Ed.). The president's conference on the examination, diagnosis and management of temporomandibular disorders. Chicago, American Dental Association, 1983. p.112-7.

Dos Santos, J. Vectorial analysis of the static equilibrium of forces generated in the mandible in centric occlusion, group function, and balanced occlusion relationships. J. Prosthet. Dent., 65:55767, 1991.

Egermark-Eriksson, I.; Carlsson, G. E. \& Magnusson, T. A long-term epidemiologic study of the relationship between occlusal factors and mandibular dysfunction in children and adolescents. J. Dent. Res., 66:67-71, 1987.

Faggion, C. M. Jr. The shortened dental arch revisited: from evidence to recommendations by the use of the GRADE approach. J. Oral Rehabil., 38:940-9, 2011.

Fueki, K.; Igarashi, Y.; Maeda, Y.; Baba, K.; Koyano, K.; Akagawa, Y.; Sasaki, K.; Kuboki, T.; Kasugai, S. \& Garrett, N. R. Factors related to prosthetic restoration in patients with shortened dental arches: a multicentre study. J. Oral Rehabil., 38:525-32, $2011 a$.

Fueki, K.; Yoshida, E. \& Igarashi, Y. A structural equation model to investigate the impact of missing occlusal units on objective masticatory function in patients with shortened dental arches. J. Oral Rehabil., 38:810-7, 2011b.

Funakoshi, M. Relations between occlusal interference and jaw muscles in response to changes in head position. J. Dent. Res., 55:684-90, 1976.

Gazit, E. \& Liebermann, M. A. Occlusal contacts following orthodontic treatment. Angle Orthod., 55:316-20, 1985.

Giardot, R. A. \& Redmond, W. R. Management and marketing - one pathway to successful orthodontic practice. J. Clin. Orthod., 39:415-8, 2005. 
Goldstein, D. F. Influence of cervical posture on mandibular movement. J. Prosthet. Dent., 52:4216, 1984.

Graham, G. S. \& Rugh, J. D. Maxillary splint occlusal guidance patterns and electromyographic activity of the jaw-closing muscles. J. Prosthet. Dent., 59:73-7, 1988.

Gysi, A. Masticating efficiency in natural and artificial teeth. Dent. Digest., 21:74-8, 1915.

Hattori, Y.; Satoh, C.; Seki, S.; Watanabe, Y.; Ogino, Y. \& Watanabe, M. Occlusal and TMJ loads in subjects with experimentally shortened dental arches. J. Dent. Res., 82:532-6, 2003.

Hellsing, G.; Hellsing, E. \& Eliasson, S. The hinge axis concept: a radiographic study of its relevance. $J$. Prosthet. Dent., 73:60-4, 1995.

Hidaka, O. \& Iwasaki, M. Influence of clenching intensity on bite force balance, occlusal contact area, and average bite pressure. J. Dent. Res., 78:1336-44, 1999.

lida, T.; Fenwick, P. B. \& loannides, A. A. Analysis of brain activity immediately before conscious teeth clenching using magnetoencephalographic method. J. Oral Rehabil., 34:487-96, 2007.

Ingervall, B. Tooth contacts of the functional and nonfunctional side in children and young adults. Arch. Oral Biol., 17:191-200, 1972.

Isaacs, D. \& Fitzgerald, D. Seven alternatives to evidence based medicine. BMJ, 18-25:1618, 1999.

Isaacson, D. A biologic concept of occlusion. J. Prevent. Dent., 3:12-6, 1976.

Ismail, A. I. \& Bader, J. D. Evidence-based dentistry in clinical practice. J. Am. Dent. Assoc., 135:78-83, 2004.

Iwase, Y.; Saitoh, I.; Okamoto, A.; Nakakura-Ohshima, K.; Inada, E.; Yamada, C.; Takemoto, Y.; Yamasaki, Y. \& Hayasaki, H. Do occlusal contact areas of maximum closing position during gum chewing and intercuspal position coincide? Arch. Oral Biol., 56:1616-23, 2011.

Jokstad, A. Methodological challenges in the study of dental occlusion. J. Oral Rehabil., 39(7):480-8, 2012.

Kanno, T. \& Carlsson, G. E. A review of the shortened dental arch concept focusing on the work by the Käyser/Nijmegen group. J. Oral Rehabil., 33:85062, 2006.

Kaplan, R. L. Concepts of occlusion. Gnathology as a basis for a concept of occlusion. Dent. Clin. North Am., 7:577-90, 1963.

Kato, T. \& Rompre, R. Sleep bruxism: and oromotor activity secondary to micro-arousal. J. Dent. Res., 80:1940-44, 2001.

Keshvad, A. \& Winstanley, R. B. An appraisal of the literature on centric relation. Part III. J. Oral Rehabil., 28:55-63, 2001.

Kinderknecht, K. E.; Wong, G. K.; Billy, E. J. \& Li, S. H. The effect of a deprogrammer on the position of the terminal transverse horizontal axis of the mandible. J. Prosthet. Dent., 28:123-31, 1992.

Koizumi, S.; Minamisawa, S.; Sasaguri, K.; Onozuka, M.; Sato, S. \& Ono, Y. Chewing reduces sympathetic nervous response to stress and prevents poststress arrhythmias in rats. Am. J. Physiol. Heart Circ. Physiol., 301:H1551-8, 2011.

Koolstra, J. H. Dynamics of the human masticatory system. Crit. Rev. Oral Biol. Med., 13:366-76, 2002.

Koyano, K.; Tsukiyama, Y. \& Kuwatsuru, R. Rehabilitation of occlusion - science or art?. J. Oral Rehabil., 39:513-21, 2012.

Lavigne, G. J.; Rempre, P. H., Poirier, G.; Huard, H.; Kato, T. \& Montplaisir, J. Y. Rhythmic masticatory muscle activity during sleep in humans. J. Dent. Res., 80:443-8, 2001.

Le Bell, Y.; Jamsa, T.; Korri, S.; Niemi, P. M. \& Alanen, $P$. Effect of artificial occlusal interferences depends on previous experience of temporomandibular disorders. Acta Odontol. Scand., 60:219-22, 2002.

Lee, T.; Saruta, J.; Sasaguri, K.; Sato, S. \& Tsukinoki, K. Allowing animals to bite reverses the effects of immobilization stress on hippocampal neurotrophin expression. Brain Res., 1195:43-9, 2008. 
Leiva, M.; Miralles, R.; Palazzi, C.; Marulanda, H.; Ormeño, G.; Valenzuela, S. \& Santander, H. Effects of laterotrusive occlusal scheme and body position on bilateral sternocleidomastoid EMG activity. Cranio, 21:99-109, 2003.

Lopez-Gavito, G.; Wallen, T. R.; Little, R. M. \& Joondeph, D. R. Anterior open-bite malocclusion: a longitudinal ten-year postretention evaluation of orthodontically treated patients. Am. J. Orthod., 87:175-86, 1985.

Lucia, V. O. Modern gnathological concepts up-dated. Chicago, Quintessence, 1983.

MacMillan, H. W. Unilateral vs bilateral balanced occlusion. J. Am. Dent. Assoc., 17:1207-20, 1930.

Manfredini, D.; Bucci, M. B.; Montagna, F. \& GuardaNardini, L. Temporomandibular disorders assessment: medicolegal considerations in the evidence-based era. J. Oral Rehabil., 38:101-19, 2011.

Manfredini, D.; Castroflorio, T.; Perinetti, G. \& GuardaNardini, L. Dental occlusion, body posture and temporomandibular disorders: where we are now and where we are heading for. J. Oral Rehabil., 39(6):463-71, 2012.

Mann, A. W. \& Pankey, L. D. Concepts of occlusion. The PM philosophy of occlusal rehabilitation. Dent. Clin. North Am., 7:621-36, 1963.

Manns, A.; Chan, C. \& Miralles, R. Influence of group function and canine guidance on electromyographic activity of elevator muscles. J. Prosthet. Dent., 57:494-501, 1987.

Manns, A. \& Diaz, G. Sistema Estomatognático. Santiago, Grafica Almagro. Universidad de Chile, 1988.

Masserman, T. A concept of jaw function with a related clinical application. J. Prosthet. Dent., 13:130-40, 1963.

McAdam, D. B. Tooth loading and cuspal guidance in canine and group function occlusions. J. Prosthet. Dent., 35:283-90, 1976.

MacDonald, J. W. C. \& Hannam, A. G. Relationship between occlusal contacts and jaw-closing muscle activity during tooth clenching. Part I. J. Prosthet. Dent., 52:718-28, 1984a.
MacDonald, J. W. C. \& Hannam, A. G. Relationship between occlusal contacts and jaw-closing muscle activity during tooth clenching. Part II. J. Prosthet. Dent., 52:862-67, 1984b.

McKee, J. R. Comparing condylar positions achieved through bimanual manipulation to condylar positions achieved through masticatory muscle contraction against an anterior deprogrammer: a pilot study. J. Prosthet. Dent., 94:389-93, 2005.

McLean, D. W. Physiologic vs pathologic occlusion. J. Am. Dent. Assoc., 25:1583-94, 1938.

McLean, L. F. Effects of changing body position on dental occlusion. J. Dent. Res., 52:1041-45, 1973.

McNamara, J. A.; Seligman, D. A. \& Okeson, J. A. Occlusion, orthodontic treatment, temporomandibular disorders: a review. J. Orofac. Pain, 9:73-90, 1995.

Michelotti, A.; Farella, M.; Gallo, L. M.; Veltri, A.; Palla, S. \& Martina, R. Effect of occlusal interference on habitual activity of human masseter. J. Dent. Res., 84:644-8, 2005.

Miles, T. S.; Nauntofte, B. \& Svensson, I. Clinical Oral Physiology. Copenhagen, Quintessence Publishing Co. Ltd., 2004.

Miralles, R.; Gallardo, F.; Baeza, M.; Valenzuela, S.; Ravera, M. J.; Ormeño, G. \& Cavada, G. Laterotrusive occlusal schemes and jaw posture tasks effects on supra- and infrahyoid EMG activity in the lateral decubitus position. Cranio, 25:10613, 2007.

Miyake, S.; Takahashi, S. S.; Yoshino, F.; Todoki, K.; Sasaguri, K.; Sato, S. \& Lee, M. C. Nitric oxide levels in rat hypothalamus are increased by restraint stress and decreased by biting. Redox Rep., 13:319, 2008.

Miyake, S.; Yoshikawa, G.; Yamada, K.; Sasaguri, K.; Yamamoto, T.; Onozuka, M. \& Sato, S. Chewing ameliorates stress-induced suppression of spatial memory by increasing glucocorticoid receptor expression in the hippocampus. Brain Res., 29:349, 2012.

Mohamed, S. E. \& Christensen, L. V. Mandibular reference position. J. Oral Rehabil., 12:355-67, 1985. 
Mohl, N. D. Diagnostic rationale: an overview. In: Mohl, N.; Zarb, G. A.; Carlsson, G. E. \& Rugh, J. D. (Eds.). A textbook of occlusion. Chicago, Quintessence, 1988. pp.179-84.

Mongini, F.; Tempia-Valent, G. \& Conserva, E. Habitual mastication in dysfunction: A computer-based analysis. J. Prosthet. Dent., 61:484-94, 1989.

Nagao, M. Comparative studies on the curve of Spee in mammals, with a discussion of its relation to the form of the fossa mandibularis. J. Dent. Res., 1:159202, 1919.

National Institutes of Health Technology Assessment Conference Statement. Management of temporomandibular disorders. J. Am. Dent. Assoc., 127:1595-606, 1996.

Nelson, S. J. \& Nowlin, T. P. Occlusion. Dent. Clin. North Am., 39:233-477, 1995.

Ohrbach, R.; List, T.; Goulet, J. P. \& Svensson, P. Recommendations from the International Consensus Workshop: convergence on an orofacial pain taxonomy. J. Oral Rehabil., 37:80712, 2010.

Okada, S., Hori, N.; Kimoto, K.; Onozuka, M.; Sato, S. \& Sasaguri, K. Effects of biting on elevation of blood pressure and other physiological responses to stress in rats: biting may reduce allostatic load. Brain Res., 1185:189-94, 2007.

Ono, Y.; Kataoka, T.; Miyake, S.; Sasaguri, K.; Sato, S. \& Onozuka, M. Chewing rescues stresssuppressed hippocampal long-term potentiation via activation of histamine $\mathrm{H} 1$ receptor. Neurosci. Res., 64:385-90, 2009.

Ono, Y.; Yamamoto, T.; Kubo, K. Y. \& Onozuka, M. Occlusion and brain function: mastication as a prevention of cognitive dysfunction. J. Oral Rehabil., 37:624-40, 2010.

Palla, S. The interface of occlusion as a reflection of conflicts within prosthodontics. Int J. Prosthodont., 18:304-6, 2005.

Pameijer, J. H.; Glickman, I. \& Roeber, F. W. Intraoral occlusal telemetry. Tooth contacts in chewing, swallowing and bruxism. J. Periodontol., 40:253-8, 1969.
Piehslinger, E.; Celar, R. M.; Horejs, T. \& Slavicek, R. Recording orthopedic jaw movements. Part IV: The rotational component during mastication. Cranio, 12:156-60, 1994.

Pokorny, P. H.; Wiens, J. P. \& Litvak, H. Occlusion for fixed prosthodontics: a historical perspective of the gnathological influence. J. Prosthet. Dent., 99:299313, 2008.

Preiskel, $\mathrm{H}$. The canine teeth related to Bennett movement. Br. Dent. J., 131:312-5, 1971.

Pullinger, A. G. \& Seligman, D. A. Quantification and validation of predictive values of occlusal variables in temporomandibular disorders using multifactorial analysis. J. Prosthet. Dent., 83:6675, 2000.

Ramirez, L. M.; Ballesteros, L. E. \& Sandoval, G. P. Tensor tympani muscle: strange chewing muscle. Med. Oral Patol. Oral Cir Bucal, 12:E96-100, 2007.

Ramirez, L. M.; Ballesteros, L. E. \& Sandoval, G. P. Topical review: temporomandibular disorders in an integral otic symptom model. Int. J. Audiol., 47:21527, 2008.

Ramirez, L. M.; Ballesteros, L. E. \& Sandoval, G. P. Tensor veli palatini and tensor tympani muscles: anatomical, functional and symptomatic links. Acta Otorrinolaringol. Esp., 61:26-33, 2010.

Reynolds, J. M. The organization of occlusion for natural teeth. J. Prosthet. Dent., 26:56-67, 1971.

Rinchuse, D. J.; Kandasamy, S. \& Sciote, J. A contemporary and evidence-based view of canine protected occlusion. Am. J. Orthod., Dentofacial Orthop., 132:90-102, 2007.

Rinchuse, D. J. \& McMinn, J. T. Summary of evidencebased systematic reviews of temporomandibular disorders. Am. J. Orthod. Dentofacial Orthop., 130:715-20, 2006.

Rinchuse, D. J. \& Sassouni, V. An evaluation of eccentric occlusal contacts in orthodontically treated subjects. Am. J. Orthod., 82:251-6, 1982.

Rinchuse, D. J. \& Sassouni, V. An evaluation of functional occlusal interferences in orthodontically treated subjects. Angle Orthod., 53:122-30, 1983. 
Rocabado, M. Physical therapy and dentistry: An overview. J. Craniomandib. Pract., 1:47-9, 19821983.

Sadowsky, C. \& BeGole, E. A. Temporomandibular joint function and functional occlusion after orthodontic treatment. Am. J. Orthod., 18:201-12, 1980.

Sadowsky, C. \& Polson, A. M. Long-term status of temporomandibular disorders and functional occlusion after orthodontic treatment: results of two long-term studies. Am. J. Orthod., 86:386-90, 1984.

Sato, C.; Sato, S.; Takashina, H.; Ishii, H.; Onozuka, M. \& Sasaguri, K. Bruxism affects stress responses in stressed rats. Clin. Oral Investig., 14:153-60, 2010.

Scaife, R. R. \& Holt, J. E. Natural occurrence of cuspid guidance. J. Prosthet. Dent., 22:225-9, 1969.

Schellas, K. P. Unstable occlusion and temporomandibular joint disease. J. Clin. Orthod., 23:332-7, 1989.

Schellhas, K. P. \& Keck, R. J. Disorders of skeletal occlusion and temporomandibular joint disease. Northwest Dent., 68:35-9, 1989.

Schuyler, C. H. Factors contributing to traumatic occlusion. J. Prosthet. Dent., 11:708-16, 1961.

Schulte, J. K.; Rooney, D. J. \& Erdman, A. G. The hinge axis transfer procedure: a three-dimensional error analysis. J. Prosthet. Dent., 51:247-51, 1984.

Schwartz, H. Occlusal variations for reconstructing the natural dentition. J. Prosthet. Dent., 55:101-5, 1986.

Senna, P.; Del Bel Cury, A. \& Rösing, C. Non-carious cervical lesions and occlusion: a systematic review of clinical studies. J. Oral Rehabil., 39(6):450-62, 2012.

Simpson, J. W.; Hesby, R. A.; Pfeifer, D. L. \& Pelleu, G. B. Jr. Arbitrary mandibular hinge axis locations. J. Prosthet. Dent., 51:819-22, 1984.

Shaw, D. M. Form and function in teeth and a rational unifying principle applied to interpretation. Int. J. Orthod., 10:703-18, 1924.

Shefter, G. J. \& McFall, W. T. Jr. Occlusal relationships and periodontal status in human adults. J. Periodontol., 55:368-74, 1984.

Shupe, R. J.; Mohamed, S. E.; Christensen, L. V.; Finger, I. M. \& Weinberg, R. Effects of occlusal guidance on jaw muscle activity. J. Prosthet. Dent., 51:811-8, 1984.

Starcke, E. N. The history of articulators: from facebows to the gnathograph, a brief history of early devices developed for recording condylar movement: part II. J. Prosthodont., 11:53-62, 2002a.

Starcke, E. N. The history of articulators: a critical history of articulators based on geometric theories of mandibular movement, part III: the "balancer" designs. J. Prosthodont., 11:305-20, 2002b.

Starcke, E. N. The history of articulators: a critical review of articulators based on geometric theories of mandibular movement, part II: Rupert Hall's conical theory. J. Prosthodont., 11:211-22, 2002c.

Starcke, E. N. The history of articulators: a critical history of articulators based on geometric theories of mandibular movement: part I. J. Prosthodont., 11:134-46, 2002d.

Starcke, E. N. The history of articulators: from facebows to the gnathograph, a brief history of early devices developed for recording condylar movement: part I. J. Prosthodont., 10:241-8, 2001.

Starcke, E. N. The history of articulators: a critical history of articulators based on "geometric" theories of mandibular movement. Part IV: Needles, Wadsworth, and a look at some who followed. J. Prosthodont., 12:51-62, 2003.

Starcke, E. N. \& Engelmeier, R. L. The history of articulators: the wonderful world of "grinders." part I. J. Prosthodont., 15:129-40, 2006.

Storey, A. T. Functional stability of orthodontic treatment: occlusion as a cause of temporomandibular disorders. In: Nanda, R. \& Burstone, C. J. (Eds.). Retention and stability in orthodontics. Philadelphia, Saunders, 1993. pp. 203-15.

Stuart, C. E. \& Golden, I. B. The history of gnathology. Ventura, CA, C.E. Stuart Gnathological Instruments, 1981. 
Stuart, C. H. \& Stallard, C. E. Diagnosis and treatment of occlusal relations of the teeth. Tex. Dent. J., 75:43035, 1957.

Stuart, C. H. \& Stallard, C. E. Concepts of occlusion-what kind of occlusion should recusped teeth be given? Dent. Clin. North Am., 7:591-600, 1963.

Tanaka, K.; Amano, N.; Satoda, T.; Murata, T.; Kawagishi, S.; Yoshino, K. \& Mizuno, N. Influences of entopeduncular nucleus stimulation upon electromyogram activity of masticatory muscles. Neuroscience, 155:669-82, 2008.

Throckmorton, G. S.; Buschang, P. H.; Hayasaki, H. \& Pinto, A. S. Changes in the masticatory cycle following treatment of posterior unilateral crossbite in children. Am. J. Orthod. Dentofacial Orthop., 120:521-9, 2001.

Tipton, R. T. \& Rinchuse, D. J. The relationship between static occlusion and functional occlusion in a dental school population. Angle Orthod., 61:57-66, 1991.

Tokiwa, O.; Park, B. K.; Takezawa, Y.; Takahashi, Y.; Sasaguri, K. \& Sato, S. Relationship of tooth grinding pattern during sleep bruxism and dental status. Cranio, 26:287-93, 2008.

Tsao, D. H. Variation of hinge interocclusal clearance and modifying mechanisms. J. Prosthet. Dent., 56:606-16, 1986.

Türp, J. C.; Greene, C. S. \& Strub, J. R. Dental occlusion: a critical reflection on past, present and future concepts. J. Oral Rehabil., 35:446-53, 2008.

Valenzuela, S.; Baeza, M.; Miralles, R.; Cavada, G.; Zúñiga, C. \& Santander, H. Laterotrusive occlusal schemes and their effect on supra- and infrahyoid electromyographic activity. Angle Orthod., 76:585-90, 2006.

van der Bilt, A. Assessment of mastication with implications for oral rehabilitation: a review. J. Oral Rehabil., 38:754-80, 2011.

Wallace, C. Management of craniomandibular disorders. Part I: A craniocervical dysfunction index. J. Orofacial Pain, 7:83-8, 1993.

Walter, M. H.; Weber, A.; Marré, B.; Gitt, I.; Gerss, J.; Hannak, W.; Range, U.; Jahn, F.; Passia, N.; Pospiech, P.; Mitov, G.; Brückner, J.; Wolfart, S.;
Busche, E.; Luthardt, R. G.; Heydecke, G. \& Marré, B. The randomized shortened dental arch study: tooth loss. J. Dent. Res., 89:818-22, 2010.

Ware, J. C. \& Rugh, J. D. Destructive bruxism: Sleep stage relationship. Sleep, 11:172-81, 1988.

Weiland, F. J. The role of occlusal discrepancies in the long-term stability of the mandibular arch. Eur. J. Orthod., 16:521-9, 1994.

Weinberg, L. A. The prevalence of tooth contact in eccentric movements of the jaws. J. Am. Dent. Assoc., 62:402-6, 1961.

Weinberg, L. A. \& Chastain, J. K. New TMJ clinical data and the implication on diagnosis and treatment. $J$. Am. Dent. Assoc., 120:305-11, 1990.

Weinberg, L. A. \& Chastain, J. K. New TMJ clinical data and the implication on diagnosis and treatment. $J$. Am. Dent. Assoc., 120:305-11, 1990.

Williamson, E. H. \& Lundquist, D. O. Anterior guidance: its effect on electromyographic activity of the temporal and masseter muscles. J. Prosthet. Dent., 49:81623, 1983.

Winstanley, R. B. The hinge-axis: a review of the literature. J. Oral Rehabil., 12:135-59, 1985.

Woda, A. A step toward setting norms: comments on the occlusal interface. Int. J. Prosthodont., 18:313-5, 2005.

Yuodelis, R. A. \& Mann, W. V. Jr. The prevalence and possible role of nonworking contacts in periodontal disease. Periodontics, 3:219-23, 1965.

Dirección para correspondencia:

Luis Miguel Ramirez Aristeguieta, DDS, MS, MSc

Profesor Asociado Facultad de Odontologia

Grupo de Investigación Estomatología Biomédica

Universidad de Antioquia

Medellin

COLOMBIA

Email: Imra3@yahoo.com

Recibido : 10-04-2012

Aceptado: 22-05-2012 